\title{
No oscillations in the Michaelis-Menten approximation of the dual futile cycle under a sequential and distributive mechanism
}

\author{
Luna Bozeman ${ }^{* 1}$ and Adriana Morales ${ }^{\dagger 2}$ \\ ${ }^{1}$ Department of Mathematical Sciences, Clemson University, Clemson, SC 29634 \\ ${ }^{2}$ Department of Mathematics, University of Puerto Rico, Río Piedras Campus, San Juan, PR 00931
}

Advisor: Anne Shiu $\ddagger \S$

\begin{abstract}
Protein phosphorylation and dephosphorylation are important intracellular processes. The main object of study in this paper is the dual futile cycle, a network that describes the dual-site phosphorylation/dephosphorylation of a protein by a kinase/phosphatase pair in a sequential and distributive mechanism. Specifically, we analyze the 2-dimensional Michaelis-Menten (M-M) approximation of this system. It has been previously shown that this system is bistable. We also know, from monotone systems theory, that every solution converges to some steady state. Here, we give a new and simpler proof of this convergence result by using Bendixon's criterion to rule out oscillations. Ultimately, understanding the behavior of this system could help us understand the original dual futile cycle (or MAPK cascades that contain it), which has recently been shown to admit oscillations via Hopf bifurcations.
\end{abstract}

\section{Introduction}

Protein phosphorylation and dephosphorylation are important intracellular processes that are involved in growth, proliferation, and cell division. These processes also play important roles in signal transduction, cell-cycle control, and nuclear signal integration [12. For example a well-known process in cellular biology is the transmission of information by signalling networks from the cell membrane to the nucleus. This in turn influences the transcription and provides the cell with a possibility of reacting to its environment. Phosphorylation is also a fundamental mechanism for altering the shape and therefore the behavior of a protein. For example signal transduction involves altering the behavior of proteins in the cascade, such as the mitogen-activated protein kinase (MAPK) cascades. Indeed, the goal of shedding light on the dynamics of this important cascade is the main motivation for this research.

Phosphorylation is the enzyme-mediated addition of a phosphate group to a protein substrate, which often modifies the function of the substrate. A multiple futile cycle (or multisite phosphorylation system) refers to when a substrate has multiple sites at which phosphate groups can be attached. Multisite phosphorylation systems can either be processive or distributive. Here, we focus on distributive systems, which occur when each enzyme-substrate binding results in only one addition or removal of a phosphate group, with 2 sites. Also, the phosphorylation/dephosphorylation system is assumed to be sequential, which occurs when the phosphate groups are added at binding sites in a certain order.

More specifically, the main object of study is the dual futile cycle, a phosphorylation system that describes the 2 -site phosphorylation of a protein by a kinase/phosphatase pair in a distributive and sequential mechanism. The structure of the dual futile cycle can be seen in Figure1 1 . For this system, Wang and Sontag showed that within restricted parameter ranges, the system exhibits generic convergence to steady states

\footnotetext{
*e-mail: lbozema@g.clemson.edu

$\dagger$ e-mail: adriana.morales@upr.edu

${ }^{\ddagger}$ Department of Mathematics, Texas A\&M University, College Station, TX 77843

$\S$ e-mail: annejls@math.tamu.edu
} 


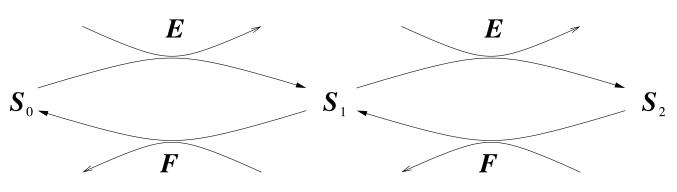

Figure 1: The dual futile cycle

but no more complicated behavior [15]. Angeli et al. showed that no species tend to be eliminated for any parameter values 1 .

Other central features of phosphorylation systems that are often analyzed are bistability and oscillations. Recently, Hell and Rendall showed that the dual futile cycle exhibits bistability for certain values of the parameters, meaning that there exist two distinct stable stationary solutions [3. But what about the oscillatory behavior of the system? Indeed, Errami et al. detected Hopf bifurcations in this system[2]. Jolley et al. considered a variant of the dual futile cycle, in which the two phosphate groups are added in the same order as they are removed (rather than the reverse order), thus, there are four phosphoforms rather than three. They showed that sustained oscillations could be observed in certain parameter regions [6]. Other variants also have been shown to admit oscillations [11, 13.

This motivates the question: Do oscillations or bistability exist in the 2-dimensional Michaelis Menten (M-M) approximation of the dual futile cycle (under a sequential and distributive mechanism)? Specifically, we are interested in the reduction of the dual futile cycle into two dimensions, using M-M theory [8]. This reduction was analyzed by Hell and Rendall to prove the bistability of the dual futile cycle [3. More specifically, for this reduction, Wang and Sontag proved that it is non-oscillatory using monotone systems theory [15. Here, we present a simpler proof of the non-oscillatory behavior of this system by using Bendixson's criterion and thus, we recover the M-M version of the result of Wang and Sontag mentioned earlier that states that every solution converges to some steady state.

As previously mentioned, one motivation for studying the behavior of the dual futile cycle is to gain more insight on the MAPK cascade system. The MAPK cascade system is a common module in many signalling networks since the MAPK cascades are central signaling pathways that regulate a wide variety of stimulated cellular processes, including proliferation, differentiation, apoptosis, and stress response. Indeed, dysregulation of these cascades is involved in the beginning and progression of diseases such as cancer, diabetes, autoimmune diseases, and developmental abnormalities [9. The MAPK cascade consists of three layers and the dual futile cycle is one of them. Numerous authors have shown numerically that the MAPK system has oscillatory behavior. Based on kinetic data on the MAPK cascades, Kholodenko predicted that the MAPK cascades can have a period of oscillations that can range from minutes to hours [7]. Quiao et al. developed a methodology for the statistical analysis of mechanistic signaling models and discovered a large region of oscillations in the Huang-Ferrell MAPK cascade model [10]. However, an analytical proof of these results does not exist [3]. Understanding the dual futile cycle can help us understand the MAPK cascade system.

The paper is organized as follows. In Section 2 we introduce the dual futile cycle. Section 3 is a detailed explanation of the reduction of the dual futile cycle into 2 dimensions using M-M theory. The core of this paper can be found in Section 4 , where we present the proof of the non-oscillatory behavior of the 2D M-M reduction of the dual futile cycle. Finally, in Section 5 we discuss some future directions for the MAPK cascade system.

\section{The dual futile cycle}

The following chemical reaction network is called the dual futile cycle which describes 2-site phosphorylation and dephosphorylation that follows a distributive and sequential mechanism: 


$$
\begin{aligned}
& S_{0}+E \underset{k_{2}}{\stackrel{k_{1}}{\rightleftarrows}} S_{0} E \stackrel{k_{3}}{\longrightarrow} S_{1}+E \underset{k_{5}}{\stackrel{k_{4}}{\rightleftarrows}} S_{1} E \stackrel{k_{6}}{\longrightarrow} S_{2}+E \\
& S_{2}+F \underset{\ell_{2}}{\stackrel{\ell_{1}}{\rightleftarrows}} S_{2} F \stackrel{\ell_{3}}{\longrightarrow} S_{1}+F \underset{\ell_{5}}{\stackrel{\ell_{4}}{\rightleftarrows}} S_{1} F \stackrel{\ell_{6}}{\longrightarrow} S_{0}+F .
\end{aligned}
$$

In this network, a substrate $S_{0}$ is converted into a product $S_{2}$ via activation reactions facilitated by an enzyme $E$. Conversely, $S_{2}$ is transformed back or deactivated into the original substrate $S_{0}$, by the action of a second enzyme $F$.

Dual futile cycles represent many types of post-translational modifications such as phosphorylation, acetylation, and methylation. For simplicity we will use the language of phosphorylation. The species in this chemical reaction network include a kinase $E$ and a phosphatase $F$. Other species include the substrates (phosphoforms) $S_{i}$ which indicate substrates with $i$ phosphate groups attached. For the dual futile cycle we have $S_{0}, S_{1}$, and $S_{2}$. The intermediate complexes, which are $S_{0} E, S_{1} E, S_{2} F$, and $S_{1} F$, are the bound enzyme-substrate complexes.

For simplicity we will change our notation. The intermediate complexes, $S_{0} E, S_{1} E, S_{2} F$, and $S_{1} F$ will now be $C_{1}, C_{2}, C_{3}$, and $C_{4}$, respectively. In this system the reaction constants $k_{i}$ and $\ell_{i}$, for $i \in\{1,2, \ldots, 6\}$, are positive numbers and the brackets indicate concentrations. According to mass-action kinetics the chemical reaction system defined by the 2-site phosphorylation network (1) can be modeled by the following ODEs:

$$
\begin{aligned}
\frac{d\left[S_{0}\right]}{d t} & =\ell_{6}\left[C_{4}\right]-k_{1}\left[S_{0}\right][E]+k_{2}\left[C_{1}\right], \\
\frac{d\left[S_{2}\right]}{d t} & =k_{6}\left[C_{2}\right]-\ell_{1}\left[S_{2}\right][F]+\ell_{2}\left[C_{3}\right], \\
\frac{d\left[C_{1}\right]}{d t} & =k_{1}\left[S_{0}\right][E]-\left(k_{2}+k_{3}\right)\left[C_{1}\right], \\
\frac{d\left[C_{2}\right]}{d t} & =k_{4}\left[S_{1}\right][E]-\left(k_{5}+k_{6}\right)\left[C_{2}\right], \\
\frac{d\left[C_{4}\right]}{d t} & =\ell_{4}\left[S_{1}\right][F]-\left(\ell_{5}+\ell_{6}\right)\left[C_{4}\right], \\
\frac{d\left[C_{3}\right]}{d t} & =\ell_{1}\left[S_{2}\right][F]-\left(\ell_{2}+\ell_{3}\right)\left[C_{3}\right], \\
\frac{d\left[S_{1}\right]}{d t} & =k_{3}\left[C_{1}\right]-k_{4}\left[S_{1}\right][E]+k_{5}\left[C_{2}\right]+\ell_{3}\left[C_{3}\right]+\ell_{5}\left[C_{4}\right]-\ell_{4}\left[S_{1}\right][F], \\
\frac{d[E]}{d t} & =\left(k_{2}+k_{3}\right)\left[C_{1}\right]+\left(k_{5}+k_{6}\right)\left[C_{2}\right]-k_{1}\left[S_{0}\right][E]-k_{4}\left[S_{1}\right][E], \\
\frac{d[F]}{d t} & =\left(\ell_{2}+\ell_{3}\right)\left[C_{3}\right]+\left(\ell_{5}+\ell_{6}\right)\left[C_{4}\right]-\ell_{1}\left[S_{2}\right][F]-\ell_{4}\left[S_{1}\right][F],
\end{aligned}
$$

together with the following conservation laws:

$$
\begin{aligned}
S_{T} & =\left[S_{0}\right]+\left[S_{2}\right]+\left[S_{1}\right]+\left[C_{1}\right]+\left[C_{2}\right]+\left[C_{4}\right]+\left[C_{3}\right], \\
E_{T} & =[E]+\left[C_{1}\right]+\left[C_{2}\right], \\
F_{T} & =[F]+\left[C_{4}\right]+\left[C_{3}\right] .
\end{aligned}
$$

The quantities in (11) are the total concentrations of the substrates and the enzymes, respectively, that are conserved under time evolution. Specifically, $S_{T}, E_{T}$, and $F_{T}$ are the total amount of concentration of the substrate, kinase, and phosphatase, respectively.

Note that the concentrations of $[E]$ and $[F]$ can be expressed in terms of $E_{T}$ and $F_{T}$ and the concentrations of their respective complexes. For example, the concentration of $[E]$ can be written as follows:

$$
\frac{d[E]}{d t}=-\frac{d\left[C_{1}\right]}{d t}-\frac{d\left[C_{2}\right]}{d t} .
$$


In a similar way, equation (8) can be expressed in terms of $S_{T}$ and equations (2) to (6). Thus it is possible to discard the equations (8) to (10), and reduce the number of equations in the system from nine to six, and the resulting system is equivalent to the original nine-equation one. This six-equation system matches the ones found in [3, 14, 15].

\section{The 2D Michaelis-Menten reduction of the dual futile cycle}

Using Michaelis-Menten (M-M) theory [8] we now reduce the network (1) to 2 dimensions. It should be noted that apart from the slightly different notation this reduction is similar to the one in [3]. The main difference is that to simplify the system even more, Hell and Rendall assume that the ratios between the constants of the reactions producing and consuming the intermediate complexes during phosphorylation are all equal [3. Here, we do not use this simplification so that our results apply to the most general M-M reduction. A similar M-M reduction can be found in [15].

To apply what is called the M-M limit the variables must be rescaled in the following way,

$$
\begin{aligned}
& E_{T}=\varepsilon \widetilde{E}_{T}, \quad\left[C_{1}\right]=\varepsilon\left[\widetilde{C}_{1}\right], \quad\left[C_{4}\right]=\varepsilon\left[\widetilde{C}_{4}\right], \quad[E]=\varepsilon[\widetilde{E}], \quad \tau=\varepsilon t, \\
& F_{T}=\varepsilon \widetilde{F}_{T}, \quad\left[C_{2}\right]=\varepsilon\left[\widetilde{C}_{2}\right], \quad\left[C_{3}\right]=\varepsilon\left[\widetilde{C}_{3}\right], \quad[F]=\varepsilon[\widetilde{F}],
\end{aligned}
$$

for a small $\varepsilon>0$ as done by 3 . The small $\varepsilon>0$ arises from the assumption that the enzyme concentrations are small compared to the concentrations of the substrates. After rescaling the concentrations and time, the new system of equations becomes

$$
\begin{aligned}
& \frac{d\left[S_{0}\right]}{d \tau}=\ell_{6}\left[\widetilde{C}_{4}\right]-k_{1}\left[S_{0}\right][\widetilde{E}]+k_{2}\left[\widetilde{C}_{1}\right], \\
& \frac{d\left[S_{2}\right]}{d \tau}=k_{6}\left[\widetilde{C}_{2}\right]-\ell_{1}\left[S_{2}\right][\widetilde{F}]+\ell_{2}\left[\widetilde{C}_{3}\right], \\
& \varepsilon \frac{d\left[\widetilde{C}_{1}\right]}{d \tau}=k_{1}\left[S_{0}\right][\widetilde{E}]-\left(k_{2}+k_{3}\right)\left[\widetilde{C}_{1}\right], \\
& \varepsilon \frac{d\left[\widetilde{C}_{2}\right]}{d \tau}=k_{4}\left[S_{1}\right][\widetilde{E}]-\left(k_{5}+k_{6}\right)\left[\widetilde{C}_{2}\right], \\
& \varepsilon \frac{d\left[\widetilde{C}_{4}\right]}{d \tau}=\ell_{4}\left[S_{1}\right][\widetilde{F}]-\left(\ell_{5}+\ell_{6}\right)\left[\widetilde{C}_{4}\right], \\
& \varepsilon \frac{d\left[\widetilde{C}_{3}\right]}{d \tau}=\ell_{1}\left[S_{2}\right][\widetilde{F}]-\left(\ell_{2}+\ell_{3}\right)\left[\widetilde{C}_{3}\right] .
\end{aligned}
$$

Next, the M-M system can be obtained as the limiting case $\varepsilon \rightarrow 0$ of the equations in 12 . Setting $\varepsilon=0$ in the system $\sqrt{12}$ gives

$$
\begin{aligned}
{\left[\widetilde{C}_{1}\right] } & =\frac{k_{1}}{k_{2}+k_{3}}\left[S_{0}\right][\widetilde{E}], \\
{\left[\widetilde{C}_{2}\right] } & =\frac{k_{4}}{k_{5}+k_{6}}\left[S_{1}\right][\widetilde{E}], \\
{\left[\widetilde{C}_{4}\right] } & =\frac{\ell_{4}}{\ell_{5}+\ell_{6}}\left[S_{1}\right][\widetilde{F}], \\
{\left[\widetilde{C}_{3}\right] } & =\frac{\ell_{1}}{\ell_{2}+\ell_{3}}\left[S_{2}\right][\widetilde{F}] .
\end{aligned}
$$

Combining 13 with two of the conservation laws in 11) yields

$$
\begin{aligned}
& \widetilde{E}_{T}=\left[1+\frac{k_{1}}{k_{2}+k_{3}}\left[S_{0}\right]+\frac{k_{4}}{k_{5}+k_{6}}\left[S_{1}\right]\right][\widetilde{E}], \\
& \widetilde{F}_{T}=\left[1+\frac{\ell_{4}}{\ell_{5}+\ell_{6}}\left[S_{1}\right]+\frac{\ell_{1}}{\ell_{2}+k_{3}}\left[S_{2}\right]\right][\widetilde{F}] .
\end{aligned}
$$


Using (13), the two remaining rescaled equations in 12 can be written as

$$
\begin{aligned}
\frac{d\left[S_{0}\right]}{d \tau} & =-\frac{k_{1} k_{3}}{k_{2}+k_{3}}\left[S_{0}\right][\widetilde{E}]+\frac{\ell_{4} \ell_{6}}{\ell_{5}+\ell_{6}}\left[S_{1}\right][\widetilde{F}], \\
\frac{d\left[S_{2}\right]}{d \tau} & =\frac{k_{4} k_{6}}{k_{5}+k_{6}}\left[S_{1}\right][\widetilde{E}]-\frac{\ell_{1} \ell_{3}}{\ell_{2}+\ell_{3}}\left[S_{2}\right][\widetilde{F}] .
\end{aligned}
$$

Note from $(11)$, that $\widetilde{S}_{T}=\left[S_{0}\right]+\left[S_{1}\right]+\left[S_{2}\right]+\varepsilon\left(\left[\widetilde{C}_{1}\right]+\left[\widetilde{C}_{2}\right]+\left[\widetilde{C}_{4}\right]+\left[\widetilde{C}_{3}\right]\right)$. Since $\widetilde{S}_{T}$ depends on $\varepsilon$, then for $\varepsilon=0$ the relation

$$
\widetilde{S}_{T}=\left[S_{0}\right]+\left[S_{1}\right]+\left[S_{2}\right]
$$

holds so that $\left[S_{1}\right]=\widetilde{S}_{T}-\left[S_{0}\right]-\left[S_{2}\right]$.

Using (14) the equations in (15) are of the form

$$
\begin{aligned}
& \frac{d\left[S_{0}\right]}{d \tau}=-\frac{a_{1}\left[S_{0}\right]}{1+b_{1}\left[S_{0}\right]+c_{1}\left[S_{1}\right]}+\frac{a_{2}\left[S_{1}\right]}{1+c_{2}\left[S_{1}\right]+d_{2}\left[S_{2}\right]}, \\
& \frac{d\left[S_{2}\right]}{d \tau}=\frac{a_{3}\left[S_{1}\right]}{1+b_{1}\left[S_{0}\right]+c_{1}\left[S_{1}\right]}-\frac{a_{4}\left[S_{2}\right]}{1+c_{2}\left[S_{1}\right]+d_{2}\left[S_{2}\right]},
\end{aligned}
$$

where

$$
\begin{aligned}
a_{1} & =\frac{k_{1} k_{3} \widetilde{E}_{T}}{k_{2}+k_{3}}, & a_{2} & =\frac{\ell_{4} \ell_{6} \widetilde{F}_{T}}{\ell_{5}+\ell_{6}}, \\
a_{3} & =\frac{k_{4} k_{6} \widetilde{E}_{T}}{k_{5}+k_{6}}, & a_{4} & =\frac{\ell_{1} \ell_{3} \widetilde{F}_{T}}{\ell_{2}+\ell_{3}}, \\
b_{1} & =\frac{k_{1}}{k_{2}+k_{3}}, & c_{1} & =\frac{k_{4}}{k_{5}+k_{6}}, \\
c_{2} & =\frac{\ell_{4}}{\ell_{5}+\ell_{6}}, & d_{2} & =\frac{\ell_{1}}{\ell_{2}+\ell_{3}},
\end{aligned}
$$

by using (14).

Finally, we substitute $\left[S_{1}\right]=\widetilde{S}_{T}-\left[S_{0}\right]-\left[S_{2}\right]$ into $[16$, which gives us our final M-M system:

$$
\begin{array}{ll}
\frac{d\left[S_{0}\right]}{d \tau}=-\frac{a_{1}\left[S_{0}\right]}{1+b_{1}\left[S_{0}\right]+c_{1}\left(\widetilde{S}_{T}-\left[S_{0}\right]-\left[S_{2}\right]\right)}+\frac{a_{2}\left(\widetilde{S}_{T}-\left[S_{0}\right]-\left[S_{2}\right]\right)}{1+c_{2}\left(\widetilde{S}_{T}-\left[S_{0}\right]-\left[S_{2}\right]\right)+d_{2}\left[S_{2}\right]} & =: f\left(\left[S_{0}\right],\left[S_{2}\right]\right), \\
\frac{d\left[S_{2}\right]}{d \tau}=\frac{a_{3}\left(\widetilde{S}_{T}-\left[S_{0}\right]-\left[S_{2}\right]\right)}{1+b_{1}\left[S_{0}\right]+c_{1}\left(\widetilde{S}_{T}-\left[S_{0}\right]-\left[S_{2}\right]\right)}-\frac{a_{4}\left[S_{2}\right]}{1+c_{2}\left(\widetilde{S}_{T}-\left[S_{0}\right]-\left[S_{2}\right]\right)+d_{2}\left[S_{2}\right]} & =: g\left(\left[S_{0}\right],\left[S_{2}\right]\right),
\end{array}
$$

where $a_{i}, b_{1}, c_{1}, c_{2}, d_{1}$, and $\widetilde{S}_{T}>0$, for $i \in\{1,2,3,4\}$.

\section{Proof of non-oscillatory behavior}

Central features of many biological and biochemical systems that are analyzed are oscillations and bistability. The bistability of the dual futile cycle, for both the full system and the 2D M-M reduction, was shown by Hell and Rendall in [3. In this section we concentrate on the oscillatory behavior of our 2D M-M reduction of the dual futile cycle (17), and we prove that it does not have periodic solutions. As explained in the introduction, this result is known from Wang and Sontag, but our proof is more simple and straightforward. To do so, we will use the well-known Bendixson's criterion.

Proposition 4.1 (Bendixson's Criterion). Suppose D is a simply connected open subset of $\mathbb{R}^{2}$. If

$$
\frac{\partial f}{\partial x}+\frac{\partial g}{\partial y} \neq 0
$$


and does not change sign in $D$, then there are no periodic orbits of the autonomous system

$$
\frac{d x}{d t}=f(x, y) \quad \text { and } \quad \frac{d y}{d t}=g(x, y)
$$

in $D$.

Theorem 4.2. The $M-M 2 D$ version of the dual futile cycle in 17 does not have periodic solutions for all choices of parameters.

Proof. We prove this by using Proposition 4.1. Consider the system (17), for some choice of $\widetilde{S}_{T}, a_{1}, a_{2}, a_{3}, a_{4}, b_{1}, c_{1}$, $c_{2}, d_{2}, \widetilde{E}_{T}, \widetilde{F}_{T} \ngtr 0$. The set $D=\left\{0 \lesseqgtr\left[S_{0}\right],\left[S_{2}\right] \lesseqgtr \widetilde{S}_{T},\left[S_{0}\right]+\left[S_{2}\right] \lesseqgtr S_{T}\right\}$ is a simply connected open subset of $\mathbb{R}^{2}$. After simplifying, the partial derivatives of system 117 are given by

$$
\begin{aligned}
\frac{\partial f}{\partial\left[S_{0}\right]} & =-\frac{a_{1}\left(1+c_{1}\left(\widetilde{S}_{T}-\left[S_{2}\right]\right)\right)}{\left(1+b_{1}\left[S_{0}\right]+c_{1}\left(\widetilde{S}_{T}-\left[S_{0}\right]-\left[S_{2}\right]\right)\right)^{2}}-\frac{a_{2}\left(1+d_{2}\left[S_{2}\right]\right)}{\left(1+c_{2}\left(\widetilde{S}_{T}-\left[S_{0}\right]-\left[S_{2}\right]\right)+d_{2}\left[S_{2}\right]\right)^{2}}, \\
\frac{\partial g}{\partial\left[S_{2}\right]} & =-\frac{a_{3}\left(1+b_{1}\left[S_{0}\right]\right)}{\left(1+b_{1}\left[S_{0}\right]+c_{1}\left(\widetilde{S}_{T}-\left[S_{0}\right]-\left[S_{2}\right]\right)\right)^{2}}+\frac{a_{4}\left(-1+c_{2}\left(-\widetilde{S}_{T}+\left[S_{0}\right]\right)\right)}{\left(1+c_{2}\left(\widetilde{S}_{T}-\left[S_{0}\right]-\left[S_{2}\right]\right)+d_{2}\left[S_{2}\right]\right)^{2}}
\end{aligned}
$$

Now, we must verify that

$$
\frac{\partial f}{\partial\left[S_{0}\right]}+\frac{\partial g}{\partial\left[S_{2}\right]} \neq 0
$$

on $D$. By adding and simplifying, we get that $\frac{\partial f}{\partial\left[S_{0}\right]}+\frac{\partial g}{\partial\left[S_{2}\right]}$ is equal to

$$
-\frac{a_{3}\left(1+b_{1}\left[S_{0}\right]\right)}{\left(1+b_{1}\left[S_{0}\right]+c_{1}\left(\widetilde{S}_{T}-\left[S_{0}\right]-\left[S_{2}\right]\right)\right)^{2}}-\frac{a_{1}\left(1+c_{1}\left(\widetilde{S}_{T}-\left[S_{2}\right]\right)\right)}{\left(1+b_{1}\left[S_{0}\right]+c_{1}\left(\widetilde{S}_{T}-\left[S_{0}\right]-\left[S_{2}\right]\right)\right)^{2}}-\frac{a_{2}\left(1+d_{2}\left[S_{2}\right]\right)+a_{4}\left(1+c_{2}\left(\widetilde{S}_{T}-\left[S_{0}\right]\right)\right)}{\left(1+c_{2}\left(\widetilde{S}_{T}-\left[S_{0}\right]-\left[S_{2}\right]\right)+d_{2}\left[S_{2}\right]\right)^{2}} .
$$

Notice that the sum in $(18)$ never changes signs because of the bounds that are set for $\left[S_{0}\right]$ and $\left[S_{2}\right]$ by the definition of $D$, and thus the sum is $<0$ on $D$. So, by Proposition 4.1, the system (17) has no periodic orbits.

Corollary 4.3. Every solution of the system (17) converges to some steady state.

Proof. The proof follows directly from the Poincaré-Bendixson theorem.

As mentioned earlier, this result was previously proven by Wang and Sontag using monotone systems theory [15].

\section{Discussion and future directions}

The proof of non-oscillatory behavior for the M-M approximation of the dual futile cycle helps us gain a better understanding of larger reaction networks. One large network that is well-studied in cell biology is the MAPK cascade network. This network describes the activity of mitogen-activated protein kinase. It contains three layers, each of which is a multiple futile cycle. More precisely, one of these layers is the dual futile cycle. The layers are linked by the fact that the fully phosphorylated form of the protein which is the substrate in one layer is the kinase for the next layer. In real biological systems the MAPK cascade is part of a larger signalling network. It is believed that one can obtain insights by first understanding the isolated cascade and later combining it with other reactions. Similarly, we can better understand the MAPK cascade by studying its component parts.

The MAPK cascade system has been modeled by a system of ODEs using mass-action kinetics by Huang and Ferrell [5]. Most recently, Hell and Rendall give a summary of what has been proved mathematically about the system of ODEs that form the MAPK cascade. They mainly focus on the issues of multistability and the existence of sustained oscillations for the system [4. Numerical and heuristic evidence has been found 
indicating that this system has periodic solutions. Quiao et al. developed a methodology for the statistical analysis of mechanistic signaling models and discovered a large region of oscillations in the Huang-Ferrell MAPK cascade model [10. Kholodenko predicted that the MAPK cascades have oscillations, which have a period that can range from minutes to hours 7 . Even though this evidence exists, there does not exist an analytical proof of oscillatory behavior in the MAPK cascade. As previously mentioned we can try to achieve the analytical proof by analyzing the oscillatory behavior of each of the layers of the MAPK cascades. This is where our analysis of the dual futile cycle comes into play.

We concluded that the M-M reduction of the dual futile cycle exhibits a non-oscillatory behavior. But one must keep in mind that in order to model the reactions of the futile dual cycle one must make assumptions about the kinetics. In many enzymatic reactions the concentrations of the enzymes are much lower than those of the corresponding substrates; this is not believed to hold in the MAPK cascades. Therefore, it is important to note that the M-M reduction is an approximation (the same one used in [3, 15]) and thus oscillations may be possible for situations when substrate concentrations are not dominant compared to the other chemical species. Thus, in cases where our assumption holds the M-M approximation is reasonable. Which makes us ask, does the non-oscillatory behavior of the M-M approximation of the dual futile cycle contradict the fact that the MAPK cascade has been said to have oscillations? Or, can it perhaps give us further insight into which parameter values the MAPK cascade can or can not yield oscillations?

\section{Acknowledgments}

We would like to first and foremost thank our mentor, Dr. Anne Shiu. This research experience would not have been possible if not for her mentorship and guidance. We would also like to thank Ola Sobieska for her assistance. This research was conducted as part of the NSF-funded REU program in Mathematics at Texas A\&M University (DMS-1460766), Summer 2016.

\section{References}

[1] D. Angeli, P. De Leenheer, and E.D. Sontag. A Petri net approach to the study of persistence in chemical reaction networks. Math. Biosci., 210(2):pp. 598-618, 2007.

[2] H. Errami, M. Eiswirth, D. Grigoriev, W.M. Seiler, T. Sturm, and A. Weber. Detection of Hopf bifurcations in chemical reaction networks using convex coordinates. J. Comput. Phys., 291:pp. 279$302,2015$.

[3] J. Hell and A.D. Rendall. A proof of bistability for the dual futile cycle. Nonlinear Anal. Real World Appl., 24:pp. 175-189, 2015.

[4] J. Hell and A.D. Rendall. Dynamical features of the MAPK cascade. ArXiv e-prints, 2015. Available at: https://arxiv.org/abs/1508.07822.

[5] C.Y. Huang and J.E. Ferrell. Ultrasensitivity in the mitogen-activated protein kinase cascade. Proc. Natl. Acad. Sci. USA, 93(19):pp. 10078-10083, 1996.

[6] C.C. Jolley, K.L. Ode, and H. R. Ueda. A design principle for a posttranslational biochemical oscillator. Cell Reports, 2(4):pp. 938-950, 2012.

[7] B.N. Kholodenko. Negative feedback and ultrasensitivity can bring about oscillations in the mitogenactivated protein kinase cascades. Eur. J. Biochem., 267(6):pp. 1583-1588, 2000.

[8] J.D. Murray. Mathematical biology, 3rd edn. Springer, New York, 2002.

[9] A. Plotnikov, E. Zehorai, S. Procaccia, and R. Seger. The mapk cascades: Signaling components, nuclear roles and mechanisms of nuclear translocation. BBA-Mol Cell Res, 1813(9):pp. 1619-1633, 2011.

[10] L. Quiao, R.B. Nachbar, I.G. Kevrekidis, and S.Y. Shvartsman. Bistability and oscillations in the Huang-Ferrell model of MAPK signaling. PLoS Comp. Biol., 3:pp. 1819-1826, 2007. 
[11] B. Y. Rubinstein, H. H. Mattingly, A. M. Berezhkovskii, and S. Y. Shvartsman. Long-term dynamics of multisite phosphorylation. Mol. Biol. Cell, 27(14):pp. 2331-2340, 2016.

[12] C. Salazar and T. Höfer. Multisite protein phosphorylation - from molecular mechanisms to kinetic models. FEBS Journal, 276(12):pp. 3177-3198, 2009.

[13] T. Suwanmajo and J. Krishnan. Mixed mechanisms of multi-site phosphorylation. J. R. Soc. Interface, 12(107):pp. 1-19, 2015.

[14] L. Wang and E.D. Sontag. On the number of steady states in a multiple futile cycle. J. Math. Bio., 57:pp. 29-52, 2008.

[15] L. Wang and E.D. Sontag. Singularly perturbed monotone systems and an application to double phosphorylation cycles. J. Nonlinear Sci., 18:pp. 527-550, 2008. 